\title{
Telescope Array Cloud Ranging Test
}

\section{Takeshi Okuda $^{a, *}$ on behalf of the Telescope Array Collaboration}

(a complete list of authors can be found at the end of the proceedings)

${ }^{a}$ Department of Physical Sciences, Ritsumeikan University, Shiga, JAPAN

E-mail: okuda@icrr.u-tokyo.ac.jp

The Telescope Array (TA) experiment detects air-showers induced by ultra high energy cosmic rays. The TA atmospheric Fluorescence telescopic Detector(TAFD) observes cosmic ray airshower, which is incident very far from the telescope. The observation does not take place in overcast night. However, the cloud status changes quickly and sometimes there are some isolated clouds. If the cloud is behind the airshower as viewed from the TAFD, the cloud presents no problem for airshower reconstruction. However if the cloud obscures the airshower, it does create a problem for airshower reconstruction. The problematic event can be rejected by airshower profile at reconstruction. However, the estimation of exposure with isolated cloud is difficult. And it should be affected more at higher energy event with relatively further from the telescope, which is lower statistics and more important for the ultra high energy cosmic ray physics. Therefore, to test the method for evaluating the correction of exposure, we installed stereo cloud cameras near one of FD sites. I report the status of the study of the Telescope Array Cloud Ranging Test.

$37^{\text {th }}$ International Cosmic Ray Conference (ICRC 2021)

July 12 th - 23rd, 2021

Online - Berlin, Germany

\footnotetext{
*Presenter
} 


\section{Introduction}

For the observation of Ultra High Energy Cosmic Ray(UHECR, Energy $>10^{19} \mathrm{eV}$ ) airshower, there is a method to use optical telescope to detect atmospheric fluorescence. This atmospheric Fluorescence telescopic Detector(FD) observes very far incident UHECR airshower to cover large area because of low statistics. FD has feature depends on atmospheric transparency and existence of cloud. The observation does not take place in overcast night. However, the cloud status changes quickly and sometimes there are some isolated clouds. If the cloud is behind the airshower as viewed from the FD, the cloud presents no problem for airshower reconstruction. However if the cloud obscures the airshower, it does create a problem for airshower reconstruction (Figure 1 Top). The problematic event can be rejected by airshower profile at reconstruction. However, the estimation of exposure with isolated cloud is difficult. And it should be affected more at higher energy event with relatively further from the telescope, which is lower statistics and more important for the UHECR physics. The Telescope Array(TA) experiment is the observatory for UHECR airshower.

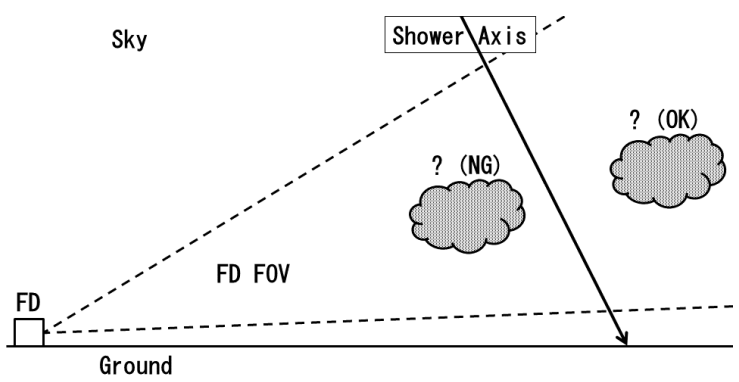

Figure 1:

Top: The conceptional image of the effect of cloud distance for shower axis

Right: FOV of TAx4FD@MDFD and Camera of TACRT

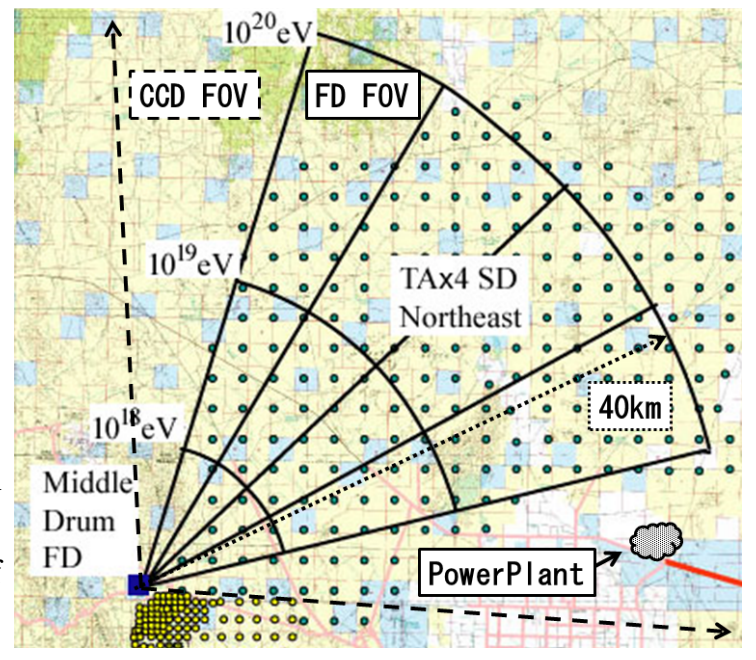

This experiment takes in Millard County, Utah, United States $\left(39.3^{\circ} \mathrm{N}, 112.9^{\circ} \mathrm{W}\right.$, altitude $\left.1382 \mathrm{~m}\right)$. The TA consists of 3 FD stations and there is expansion plan named TAx 4 for higher energy than TA. One of the TAx4FD is located Middle Drum FD(MDFD) station which is the north FD station of TA (Figure 1 Right). As written above, because the target airshower for TAx4FD is relatively further from telescope, the existence of cloud may affect estimation of exposure. There are several methods to measure cloud distance. The lidar field of view is very narrow. It cannot cover area whose cloud status changes quickly. Radar is too expensive and large power consumption. The active method like above two is not appropriate to coexist with FD observation. Therefore as passive method, Telescope Array Cloud Ranging Test(TACRT) has started to test the method for evaluating the correction of exposure. Stand alone stereo cloud cameras was installed near MDFD.

\section{Instruments}

Camera of TACRT is $1 / 3$ CCD controlled by USB2.0 with large angle DC Iris lens. The camera and heater for dew and frost are enclosed in outdoor case with wind protector. Azimuth direction 
of TACRT is set to the center of azimuth of field of view of TAx4FD (Figure 1 Right). The field of view of camera is about $95^{\circ}$ horizontally. This angle much wider than TAx4FD is to get the North Star and the sky above thermal power plant simultaneously. This is for adjusting camera and calibrating distance. The camera has monochrome 8 bit $1024 \times 768$ pixels. Therefore, the horizontal resolution is roughly $0.1^{\circ}$ per pixel. In addition to camera, there is thermohygrometer, dewsensor, GPS, and PC for data acquisition with $2.4 \mathrm{GHz}$ wireless LAN module and plane antenna. TACRT is operated automatically as stand alone, powered by solar(450W) battery(12V600Ah) system. Automatic data transfer and occasional control is done via TA long distance wireless network. Two CRT system located about $0.9 \mathrm{~km}$ apart perpendicular to the the center of azimuth of field of view of TAx4FD and CRT. This configuration correspond to about 12 pixels shift on image for $40 \mathrm{~km}$ distance (Figure 1 Right), which is enough to test the method. Left eye named SCa is located inside of MDFD station, Right eye named SCb is located in the field (Figure 2). Example of monitoring plot near the winter solstice is shown on Figure 3. It is from 3 days data including 1 snowy day. Solar battery system supplies enough power for stable operation. GPS is used location measurement and check stability of PC clock. Brightness of sky changes a lot and quickly depending of sun

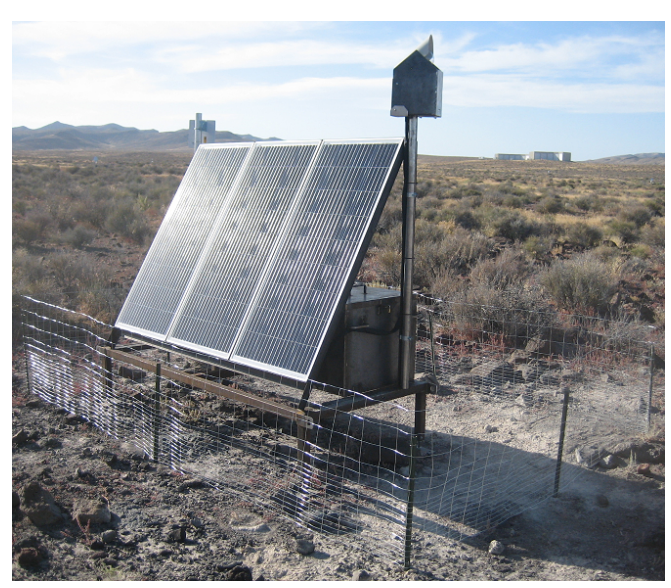

Figure 2: View from $\mathrm{SCb}$ to MDFD

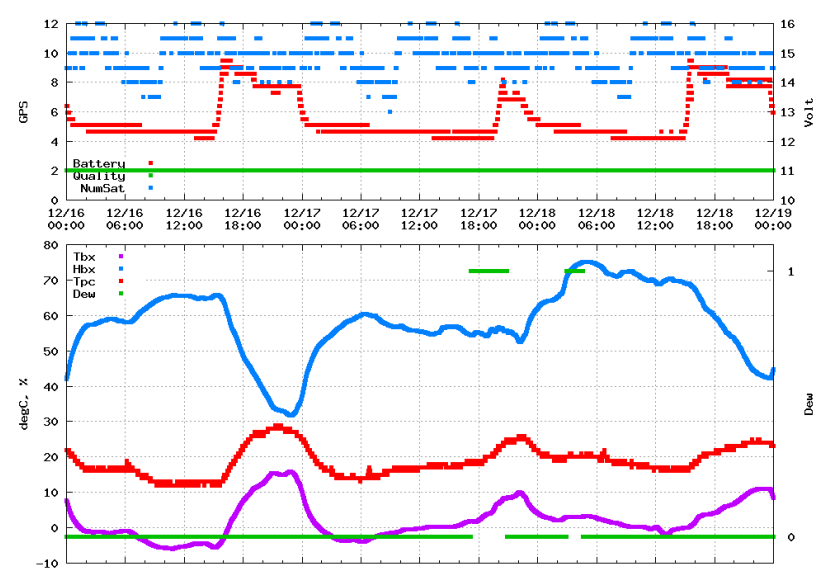

Figure 3: Monitoring plot near the winter solstice

height. Therefore, the camera setting for capturing is changed by multiple step. Changing setting is Iris=Open/Close, Gain=H/L and Exposure. Closed iris is 1/90000 of light compared to Open. Gamma is also changeable but fixed to 1 . Total exposure is always $20 \mathrm{~s}, 80$ frame of $0.25 \mathrm{~s}$ in daytime and 5 frame of $4 \mathrm{~s}$. The transition is during nautical twilight. All frame data read out from $\mathrm{CCD}$ is stacked in binary on memory. At night, closed iris data is also stacked in binary on memory as dark frame. After subtraction of dark frame, binary data is scaled to desired exposure. At this phase, the maximum ADC value is larger than 255. For the storage and data transfer, final format is 8 bit bmp. For each pixel, the ADC value more than 100 scale linearly to set $255 \times$ stacked frame in binary to 255 . Therefore, high sensitive pixels and somewhat large dynamic range pixels coexist in one image. These capturing is done every 1 minute, simultaneously at SCa and SCb. However in daytime, to suppress data size, storing image is every 10 minute. 


\section{Observation}

The compressed sky image is transferred to WWW every 10 minute to check sky from anywhere online before FD observation (Figure 4). In night time, sky image is transferred to TAx4FD@MDFD PC every 1 minute to support FD observation (Figure 5). Example of stereo sky image is shown

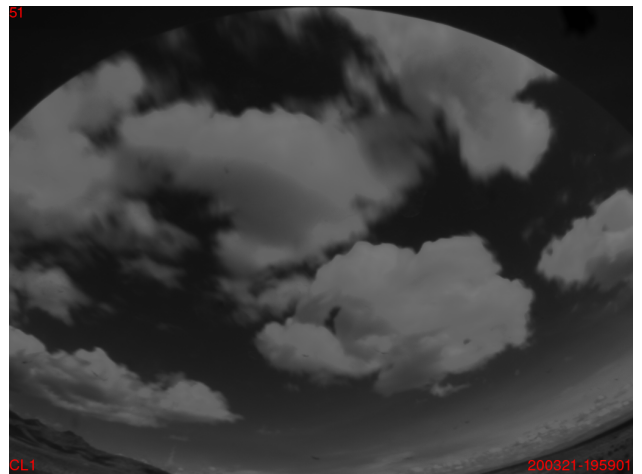

Figure 4: daytime sky image

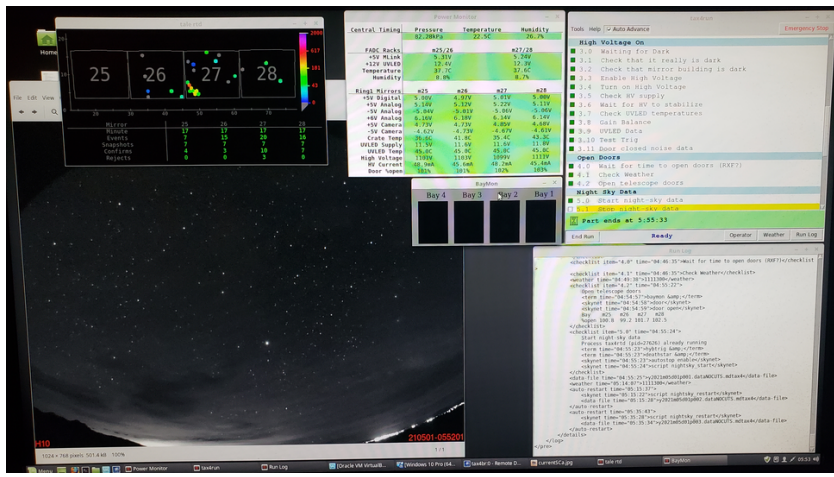

Figure 5: TAx4FD@MDFD control monitor

on Figure 6. Cloud at FD site is darker than sky. Because of this, it is difficult to get cloud image directly. Cloud distance can be evaluated by the visibility of the background star. As written previously, TACRT does not care overcast night. The target is isolated cloud in clear night, in which FD shower analysis is usually executed. The parallax of the cloud can be seen on Figure 6 . And the difference of the background star visibility also can be seen.

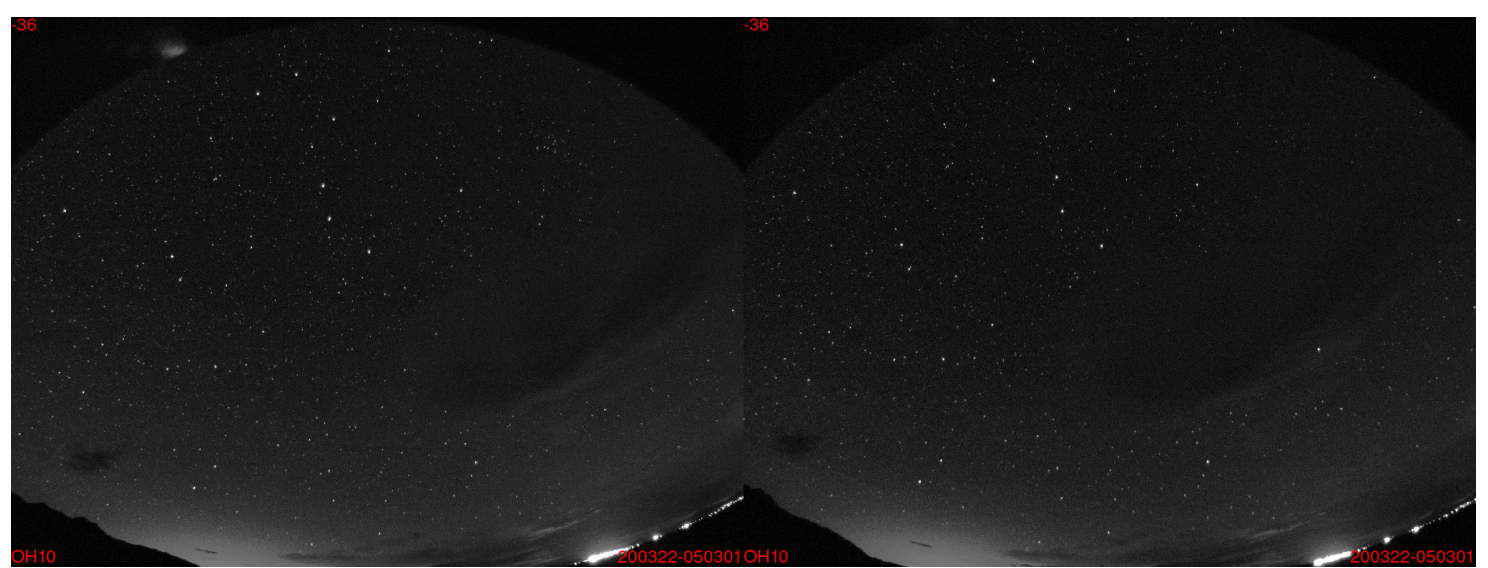

Figure 6: Simultaneous sky image by $\mathrm{SCa}$ and $\mathrm{SCb}$ with isolated cloud.

\section{Analysis}

The first step of analysis is to determine the direction of each pixels. The very clear night image is used for this. Catalog star position and detected star position will be homologized. Camera direction and rotation and distortion will be evaluated. Then, judging the difference of star visibility 
between stereo sky image. Example of detected star position is shown on Figure 7. Even bright star, the difference of star visibility around isolated cloud can be seen.
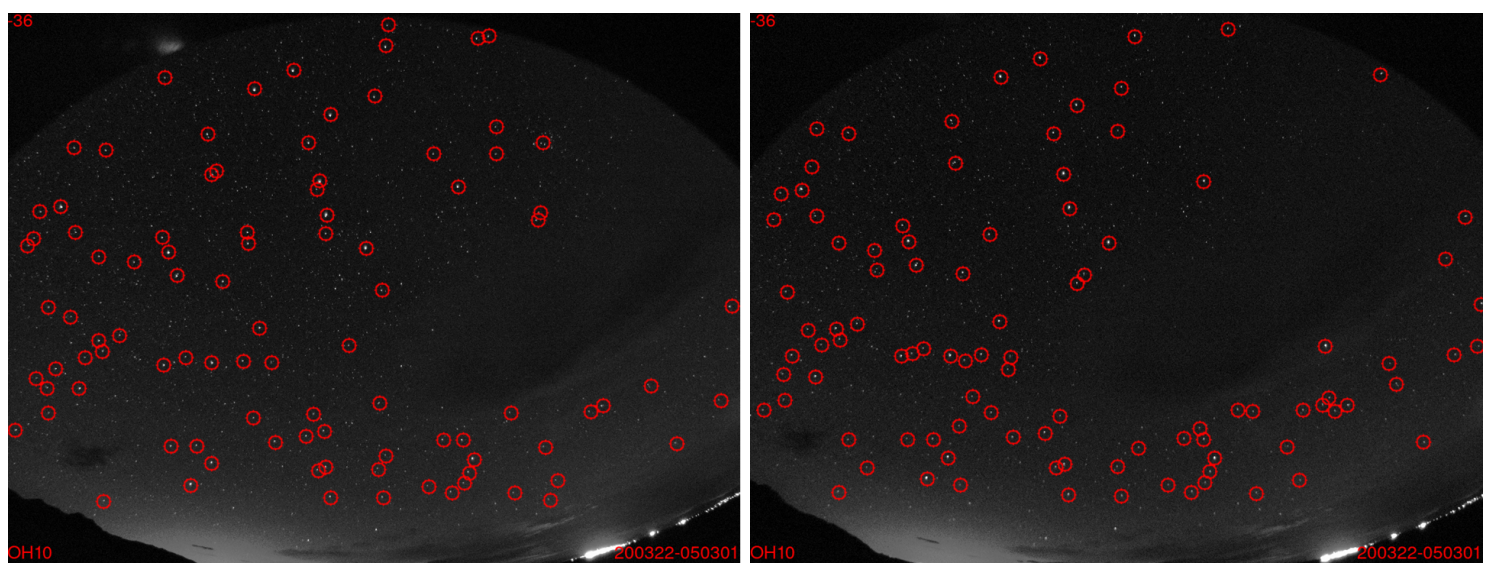

Figure 7: Simultaneous sky image by $\mathrm{SCa}$ and $\mathrm{SCb}$ with isolated cloud. Red circle shows bright star position.

\section{Summary and Plan}

TACRT is test of the method to measure cloud distance for evaluating the correction of exposure. However, it also works as support quasi remote operation under pandemic.

The analysis of stereo image is on going.

Two CRT system observe cloud distance uniquely. Uncertainty of cloud distance cannot be evaluated by this system. If there is the third CRT, different pair of CRT supplies cross calibration. The materials for the third CRT is already stored near observation site. The third CRT will be installed in the future depending on various circumstances.

\section{Acknowledgments}

TACRT is supported mainly by JSPS KAKENHI Grant Number JP19K03895. In addition, TACRT is also supported by the joint research program of the Institute for Cosmic Ray Research of University of Tokyo and the research support program of Ritsumeikan University. TACRT gets cooperation with Telescope Array experiment, especially University of Utah. The experimental site became available through the cooperation of the Utah School and Institutional Trust Lands Administration (SITLA). 


\section{Full Authors List: Telescope Array Collaboration}

R.U. Abbasi ${ }^{1}$, M. Abe ${ }^{2}$, T. Abu-Zayyad ${ }^{1,3}$, M. Allen ${ }^{3}$, Y. Arai ${ }^{4}$, E. Barcikowski ${ }^{3}$, J.W. Belz ${ }^{3}$, D.R. Bergman ${ }^{3}$, S.A. Blake ${ }^{3}$, I. Buckland ${ }^{3}$, R. $\mathrm{Cady}^{3}$, B.G. Cheon ${ }^{5}$, J. Chiba ${ }^{6}$, M. Chikawa ${ }^{7}$, T. Fujii ${ }^{8}$, K. Fujisue ${ }^{7}$, K. Fujita ${ }^{4}$, R. Fujiwara ${ }^{4}$, M. Fukushima ${ }^{7,9}$, R. Fukushima ${ }^{4}$, G. Furlich ${ }^{3}$, R. Gonzalez ${ }^{3}$, W. Hanlon ${ }^{3}$, M. Hayashi ${ }^{10}$, N. Hayashida ${ }^{11}$, K. Hibino ${ }^{11}$, R. Higuchi ${ }^{7}$, K. Honda $^{12}$, D. Ikeda ${ }^{11}$, T. Inadomi ${ }^{13}$, N. Inoue ${ }^{2}$, T. Ishii ${ }^{12}$, H. Ito ${ }^{14}$, D. Ivanov ${ }^{3}$, H. Iwakura ${ }^{13}$, H.M. Jeong ${ }^{15}$, S. Jeong ${ }^{15}$, C.C.H. Jui ${ }^{3}$, K. Kadota ${ }^{16}$, F. Kakimoto ${ }^{11}$, O. Kalashev ${ }^{17}$, K. Kasahara ${ }^{18}$, S. Kasami ${ }^{19}$, H. Kawai ${ }^{20}$, S. Kawakami ${ }^{4}$, S. Kawana ${ }^{2}$, K. Kawata ${ }^{7}$, E. Kido ${ }^{14}$, H.B. Kim ${ }^{5}$, J.H. Kim ${ }^{3}$, J.H. Kim ${ }^{3}$, M.H. Kim ${ }^{15}$, S.W. Kim ${ }^{15}$, Y. Kimura ${ }^{4}$, S. Kishigami ${ }^{4}$, Y. Kubota ${ }^{13}$, S. Kurisu ${ }^{13}$, V. Kuzmin ${ }^{17}$, M. Kuznetsov ${ }^{17,21}$, Y.J. Kwon ${ }^{22}$, K.H. Lee ${ }^{15}$, B. Lubsandorzhiev ${ }^{17}$, J.P. Lundquist ${ }^{3,23}$, K. Machida ${ }^{12}$, H. Matsumiya ${ }^{4}$, T. Matsuyama ${ }^{4}$, J.N. Matthews ${ }^{3}$, R. Mayta ${ }^{4}$, M. Minamino ${ }^{4}$, K. Mukai $^{12}$, I. Myers ${ }^{3}$,

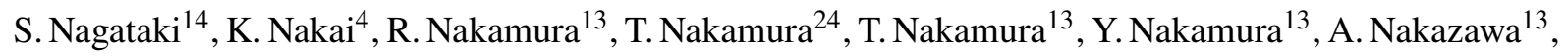

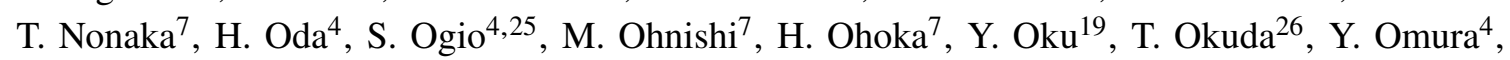
M. Ono ${ }^{14}$, R. Onogi ${ }^{4}$, A. Oshima ${ }^{4}$, S. Ozawa ${ }^{27}$, I.H. Park ${ }^{15}$, M. Potts ${ }^{3}$, M.S. Pshirkov ${ }^{17,28}$, J. Remington ${ }^{3}$, D.C. Rodriguez ${ }^{3}$, G.I. Rubtsov ${ }^{17}$, D. Ryu ${ }^{29}$, H. Sagawa ${ }^{7}$, R. Sahara ${ }^{4}$, Y. Saito ${ }^{13}$, N. Sakaki ${ }^{7}$, T. Sako ${ }^{7}$, N. Sakurai ${ }^{4}$, K. Sano ${ }^{13}$, K. Sato ${ }^{4}$, T. Seki ${ }^{13}$, K. Sekino ${ }^{7}$, P.D. Shah ${ }^{3}$, Y. Shibasaki ${ }^{13}$, F. Shibata ${ }^{12}$, N. Shibata ${ }^{19}$, T. Shibata ${ }^{7}$, H. Shimodaira ${ }^{7}$, B.K. Shin ${ }^{29}$, H.S. Shin ${ }^{7}$, D. Shinto ${ }^{19}$, J.D. Smith ${ }^{3}$, P. Sokolsky ${ }^{3}$, N. Sone ${ }^{13}$, B.T. Stokes ${ }^{3}$, T.A. Stroman ${ }^{3}$, T. Suzawa ${ }^{2}$, Y. Takagi ${ }^{4}$, Y. Takahashi ${ }^{4}$, M. Takamura ${ }^{6}$, M. Takeda ${ }^{7}$, R. Takeishi ${ }^{7}$, A. Taketa $^{30}$, M. Takita $^{7}$, Y. Tameda ${ }^{19}$, H. Tanaka ${ }^{4}$, K. Tanaka ${ }^{31}$, M. Tanaka ${ }^{32}$, Y. Tanoue ${ }^{4}$, S.B. Thomas ${ }^{3}$, G.B. Thomson ${ }^{3}$,

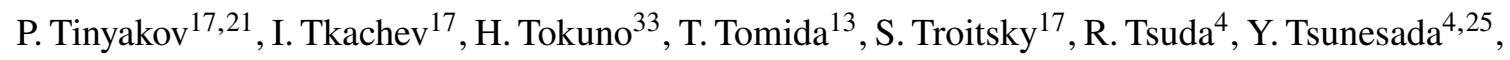

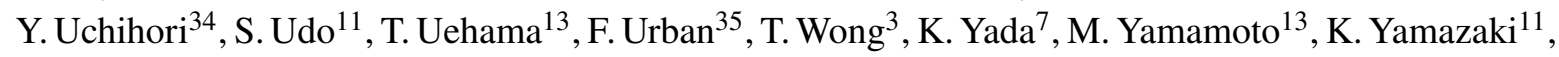
J. Yang ${ }^{36}$, K. Yashiro ${ }^{6}$, F. Yoshida ${ }^{19}$, Y. Yoshioka ${ }^{13}$, Y. Zhezher ${ }^{7,17}$, and Z. Zundel ${ }^{3}$

${ }^{1}$ Department of Physics, Loyola University Chicago, Chicago, Illinois, USA

2 The Graduate School of Science and Engineering, Saitama University, Saitama, Saitama, Japan

${ }^{3}$ High Energy Astrophysics Institute and Department of Physics and Astronomy, University of Utah, Salt Lake City, Utah, USA

${ }^{4}$ Graduate School of Science, Osaka City University, Osaka, Osaka, Japan

5 Department of Physics and The Research Institute of Natural Science, Hanyang University, Seongdong-gu, Seoul, Korea

${ }^{6}$ Department of Physics, Tokyo University of Science, Noda, Chiba, Japan

${ }^{7}$ Institute for Cosmic Ray Research, University of Tokyo, Kashiwa, Chiba, Japan

${ }^{8}$ The Hakubi Center for Advanced Research and Graduate School of Science, Kyoto University, Kitashirakawa-Oiwakecho, Sakyo-ku, Kyoto, Japan

${ }^{9}$ Kavli Institute for the Physics and Mathematics of the Universe (WPI), Todai Institutes for Advanced Study, University of Tokyo, Kashiwa, Chiba, Japan

${ }^{10}$ Information Engineering Graduate School of Science and Technology, Shinshu University, Nagano, Nagano, Japan

${ }^{11}$ Faculty of Engineering, Kanagawa University, Yokohama, Kanagawa, Japan

${ }^{12}$ Interdisciplinary Graduate School of Medicine and Engineering, University of Yamanashi, Kofu, Yamanashi, Japan 
${ }^{13}$ Academic Assembly School of Science and Technology Institute of Engineering, Shinshu University, Nagano, Nagano, Japan

${ }^{14}$ Astrophysical Big Bang Laboratory, RIKEN, Wako, Saitama, Japan

${ }^{15}$ Department of Physics, Sungkyunkwan University, Jang-an-gu, Suwon, Korea

${ }^{16}$ Department of Physics, Tokyo City University, Setagaya-ku, Tokyo, Japan

${ }^{17}$ Institute for Nuclear Research of the Russian Academy of Sciences, Moscow, Russia

${ }^{18}$ Faculty of Systems Engineering and Science, Shibaura Institute of Technology, Minato-ku, Tokyo, Japan

${ }^{19}$ Department of Engineering Science, Faculty of Engineering, Osaka Electro-Communication University, Neyagawa-shi, Osaka, Japan

${ }^{20}$ Department of Physics, Chiba University, Chiba, Chiba, Japan

${ }^{21}$ Service de Physique Théorique, Université Libre de Bruxelles, Brussels, Belgium

${ }^{22}$ Department of Physics, Yonsei University, Seodaemun-gu, Seoul, Korea

${ }^{23}$ Center for Astrophysics and Cosmology, University of Nova Gorica, Nova Gorica, Slovenia

${ }^{24}$ Faculty of Science, Kochi University, Kochi, Kochi, Japan

${ }^{25}$ Nambu Yoichiro Institute of Theoretical and Experimental Physics, Osaka City University, Osaka, Osaka, Japan

${ }^{26}$ Department of Physical Sciences, Ritsumeikan University, Kusatsu, Shiga, Japan

${ }^{27}$ Quantum ICT Advanced Development Center, National Institute for Information and Communications Technology, Koganei, Tokyo, Japan

${ }^{28}$ Sternberg Astronomical Institute, Moscow M.V. Lomonosov State University, Moscow, Russia

${ }^{29}$ Department of Physics, School of Natural Sciences, Ulsan National Institute of Science and Technology, UNIST-gil, Ulsan, Korea

${ }^{30}$ Earthquake Research Institute, University of Tokyo, Bunkyo-ku, Tokyo, Japan

${ }^{31}$ Graduate School of Information Sciences, Hiroshima City University, Hiroshima, Hiroshima, Japan

${ }^{32}$ Institute of Particle and Nuclear Studies, KEK, Tsukuba, Ibaraki, Japan

${ }^{33}$ Graduate School of Science and Engineering, Tokyo Institute of Technology, Meguro, Tokyo, Japan

${ }^{34}$ Department of Research Planning and Promotion, Quantum Medical Science Directorate, National Institutes for Quantum and Radiological Science and Technology, Chiba, Chiba, Japan

${ }^{35}$ CEICO, Institute of Physics, Czech Academy of Sciences, Prague, Czech Republic

${ }^{36}$ Department of Physics and Institute for the Early Universe, Ewha Womans University, Seodaaemungu, Seoul, Korea 